\title{
Venous outflow congestion is related to poor recurrence-free survival of living donor liver transplantation recipients with hepatocellular carcinoma
}

Jinsoo Rhu

Division of Transplantation, Department of Surgery, Samsung Medical Center, Seoul, Korea

Background: This study analyzed the impact of venous outflow congestion in the liver graft on hepatocellular carcinoma recurrence in liver transplantation recipients.

Methods: Hepatocellular carcinoma patients who underwent living donor liver transplantation at Samsung Medical Center between 2007 and 2018 were included. The congested volume was calculated based on 2-week post-transplantation computed tomography. Recurrence-free survival and overall survival were analyzed using the multivariable Cox proportional hazard model including the degree of venous congestion.

Results: A total of 582 patients were included. There were 232 patients (39.9\%) with certain degree of congestion volume. Kaplan-Meier survival analyses showed 1-, 5-, and 10-year recurrence-free survivals of $86.0 \%, 72.2 \%$, and $70.7 \%$, respectively, and overall survivals of $91.5 \%, 73.4 \%$, and $68.9 \%$, respectively. While congestion volume per $10 \mathrm{~cm} 3$ was a significant risk factor for recurrence-free survival (hazard ratio [HR], 1.021; 95\% confidence interval [Cl], 1.005-1.038; $P=0.010$ ), there was no significant relationship with overall survival $(\mathrm{HR}, 1.012 ; 95 \% \mathrm{Cl}, 0.997-1.028 ; \mathrm{P}=0.111)$.

Conclusions: Venous outflow congestion in the liver after living donor liver transplantation was related to the poor recurrence-free survival of hepatocellular carcinoma patients.

Corresponding author: Jinsoo Rhu

E-mail: jsrrules@gmail.com

(C) The Korean Society for Transplantation

This is an Open Access article distributed under the terms of the Creative Commons Attribution Non-Commercial License (http://creativecommons.org/licenses/by-nc/4.0/) which permits unrestricted non-commercial use, distribution, and reproduction in any medium, provided the original work is properly cited. 